\section{Physical inactivity: the biggest public health problem of the 21st century}

\author{
Steven N Blair
}

There is now overwhelming evidence that regular physical activity has important and wide-ranging health benefits. These range from reduced risk of chronic diseases such as heart disease, type 2 diabetes, and some cancers to enhanced function and preservation of function with age. As a member of the geriatric set, I am personally delighted that there is strong emerging evidence that activity delays cognitive decline and is good for brain health as well as having extensive benefits for the rest of the body.

I believe that evidence supports the conclusion that physical inactivity is one of the most important public health problems of the 21st century, and may even be the most important. This is not to deny the relevance of other health issues; and certainly we need to pay much more attention to healthful eating habits, smoking prevention and cessation programmes, and state-of-the art and evidence-based preventive medical care. My overriding concern is that the crucial importance of physical activity is undervalued and underappreciated by many individuals in public health and clinical medicine. Figure 1 presents data that have helped lead me to these conclusions about the importance of inactivity. As I suspect most of you are aware, the attributable fraction is an estimate of the number of deaths in a population that would have been avoided if a specific risk factor had been absent. That is, if all smokers were non-smokers or all inactive persons were getting 30 minutes of walking on at least 5 days of the week. The data in figure 1 are based on follow-up of a large population of women and men in the Aerobics Center Longitudinal Study (ACLS). Attributable fraction estimates depend on the strength of association between an exposure and an outcome, and also on the prevalence of that particular risk factor in the population. Figure 1 shows

Correspondence to: Professor Steven N Blair, Department of Exercise Science and Epidemiology/ Biostatistics, Arnold School of Public Health, University of South Carolina, South Carolina, USA;

sblair@gwm.sc.edu that low cardiorespiratory fitness accounts for about $16 \%$ of all deaths in both women and men in this population, and this is substantially more, with the exception of hypertension in men, than the other risk factors. I ask you to consider how often each of these risk factors is evaluated in a typical medical examination, and how often each risk factor is treated if found to be elevated. I have no data, but I wager that the typical physician is $10-50$ times more likely to measure cholesterol, blood pressure, and BMI than to measure fitness. Fitness was measured in the ACLS with a maximal exercise test on a treadmill, so this exposure is based on an objective, laboratory measurement. Of course such an exercise test may not be feasible in many clinical examinations, but physicians and other clinicians could at least take a physical activity history and put physical activity on the patient's agenda.

Another example of the importance of fitness is illustrated in figure 2. In this study we followed 2316 men with documented type 2 diabetes for an average of 15.9 years, during which time 179 of them died of cardiovascular disease (CVD). The

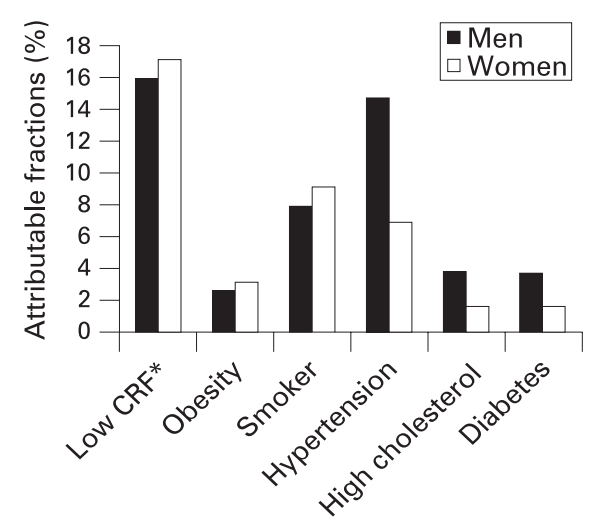

Figure 1 Attributable fractions (\%) for allcause deaths in 40842 (3333 deaths) men and 12943 (491 deaths) women in the Aerobics Center Longitudinal Study. The attributable fractions are adjusted for age and each other item in the figure. ${ }^{*}$ Cardiorespiratory fitness determined by a maximal exercise test on a treadmill. data in figure 2 clearly show a strong inverse gradient for CVD death across fitness categories within each BMI category. Note that the obese men who were moderately/highly fit had less than half the risk of dying than the normal-weight men who were unfit. Every day tens of thousands of patients with type 2 diabetes attend a medical evaluation. How many of these patients have their height and weight measured and their BMI calculated, after which they are assigned to either the normal weight, overweight, or obese category? Conversely, how many of these patients have their cardiorespiratory fitness evaluated, or even have a careful and accurate physical activity history obtained? My guess is that if the physician mentions physical activity it may be "I think you should take up some exercise, it will help you lose weight". This is the wrong message. Of course regular physical activity will help with weight management, but the activity will be very important to the patient's health, whether or not they lose weight.

As you can tell, I am passionate about the importance of regular physical activity for individuals' and the public's health, and think we must focus much more attention on this issue. Therefore I was delighted when Professor Khan invited me to help coordinate a special issue of the British Journal of Sports Medicine to focus on the topic. He and I have worked over the past few months to recruit an outstanding group of scientists

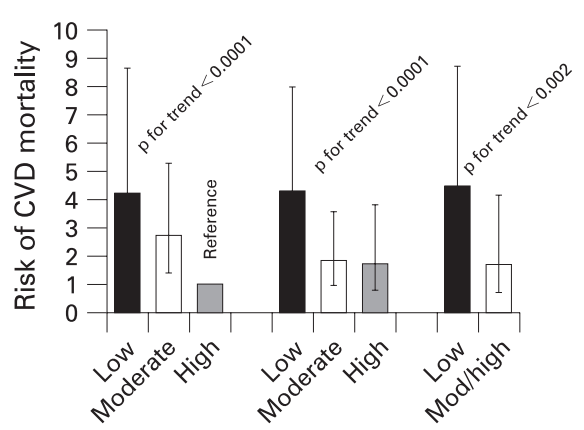

$18.5<\mathrm{BMl}<25.0 \quad 25.0 \leqslant \mathrm{BMl}<30.030 .0 \leqslant \mathrm{BMl}<35.0$

Figure 2 Risk of cardiovascular disease mortality by cardiorespiratory fitness and body mass index categories, 2316 men with type 2 diabetes at baseline, 179 deaths. Risk ratios are adjusted for age and examination year. Black bars $=$ low cardiorespiratory fitness, white bars $=$ moderate cardiorespiratory fitness (in the obese category, the white bar includes both moderate and high cardiorespiratory fitness, and the grey bar = high cardiorespiratory fitness). (Adapted from Church TS et al. Arch Int Med 2005;165:2114-20) 
and clinicians to prepare special reports on various aspects of physical activity and health. I am grateful that many of the top people in exercise science from around the world agreed to help with this special issue, and I am very pleased with the high-quality and informative reports that they have provided. This issue contains 18 articles on many topics that provide the background and rationale for giving more attention to physical activity in clinical and public health settings. If you peruse the Table of Contents I am confident that you will find topics of interest. Then the February issue will contain more outstanding reports from leading investigators, with a focus on physical activity interventions in a variety of settings.

Competing interests: None. 